\title{
Molybdenum-Promoted Synthesis of Isoquinuclidines \\ with Bridgehead CF3 Groups
}

Justin H. Wilde, Diane A. Dickie, W. Dean Harman Department of Chemistry, University of Virginia, Charlottesville, VA 22904

\section{Abstract}

Although nitrogen-coordinated complexes of pyridine are ubiquitous in organometallic chemistry, other coordination modes are substantially less common. Especially unusual are $\eta^{2}-(C, C)$-coordinated complexes, with only a few examples found in the literature. Dihapto-coordination is of considerable interest, as this binding mode promotes addition reactions that effect dearomatization. This work explores the synthesis and organic reactivity of dihapto-bound pyridine complexes of a second-row metal, molybdenum, with the goal of producing substituted di- and tetrahydropyridines via regioand stereoselective additions. Given that molybdenum generally forms weaker bonds to arenes compared to analogous third-row dearomatization agents, it was uncertain whether such $\eta^{2}$-pyridine complexes could be successfully isolated and carried through organic transformations. MoTp(NO)(DMAP)(4,5-n $n^{2}$-2-(trifluoromethyl)pyridine) was investigated to elucidate the effect of an electron-withdrawing substituent on dihaptoelucidate the effect of an electron-withdrawing substituent on dihaptonucleophiles, including Grignard reagents and organozinc compounds, allows the formation of various substituted dihydropyridine complexes. The free dihydropyridines can be liberated from the metal by oxidation, and elaborated to novel bridgehead $\mathrm{CF}_{3}$-substituted isoquinuclidines. An enantioselective variant has been demonstrated, illustrating the synthetic utility of this method to access compounds of potential medicinal relevance.

\section{Dearomatization}

Aromatics are inexpensive, readily available, and offer multiple unsaturated sites for functionalization

Dihapto coordination by $\pi$-basic metal complex disrupts aromaticity via $\mathrm{d} \pi$ to $\pi^{*}$ electron donation

Aromatic is activated toward subsequent stereo- and regioselective electrophilic, nucleophilic additions

等]一

\section{Pyridine Complexes via Ligand Exchange}

Stereoelectronic effects of an appropriate substituent circumvent $\mathrm{kN}$ coordination, the favored coordination mode for unsubstituted pyridine
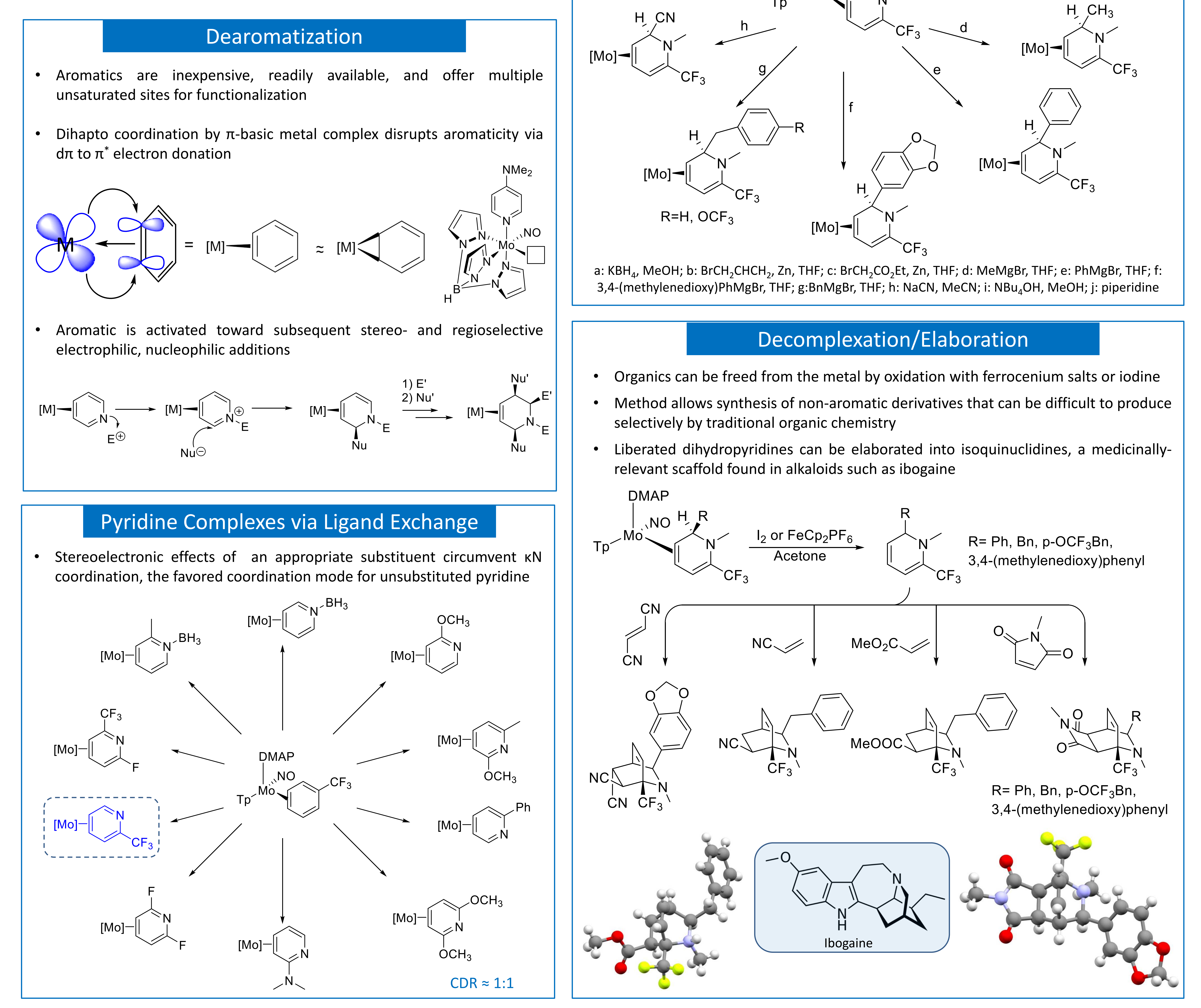

\section{Organic Reactivity}

- The 4,5- $\eta^{2}-2$-(trifluoromethyl)pyridine complex can be trapped in the solid state as a single coordination diastereomer and subsequently $\mathrm{N}$-alkylated

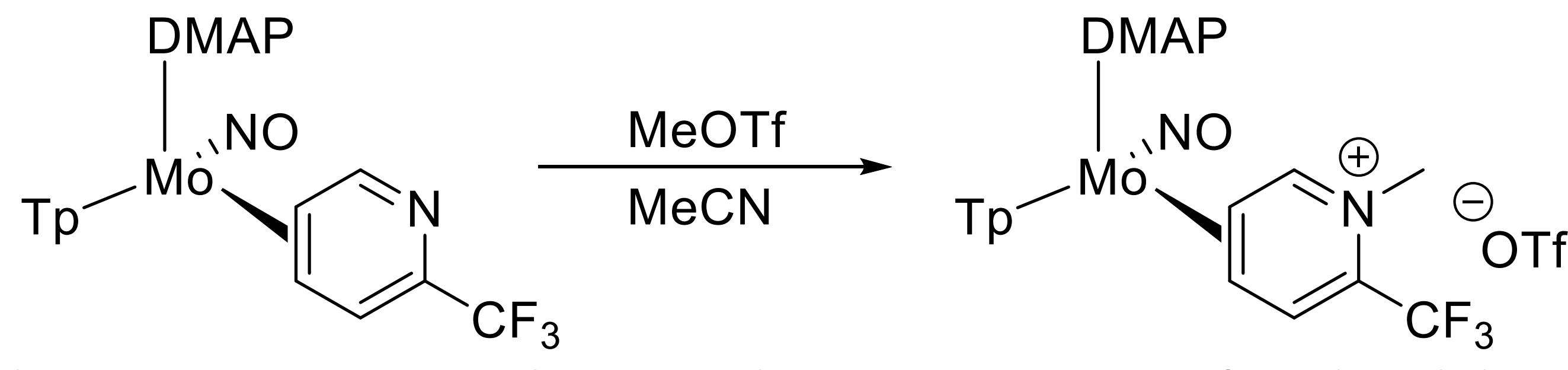

The resulting pyridinium complex can take part in a range of nucleophilic additions, which proceed stereo- and regioselectively

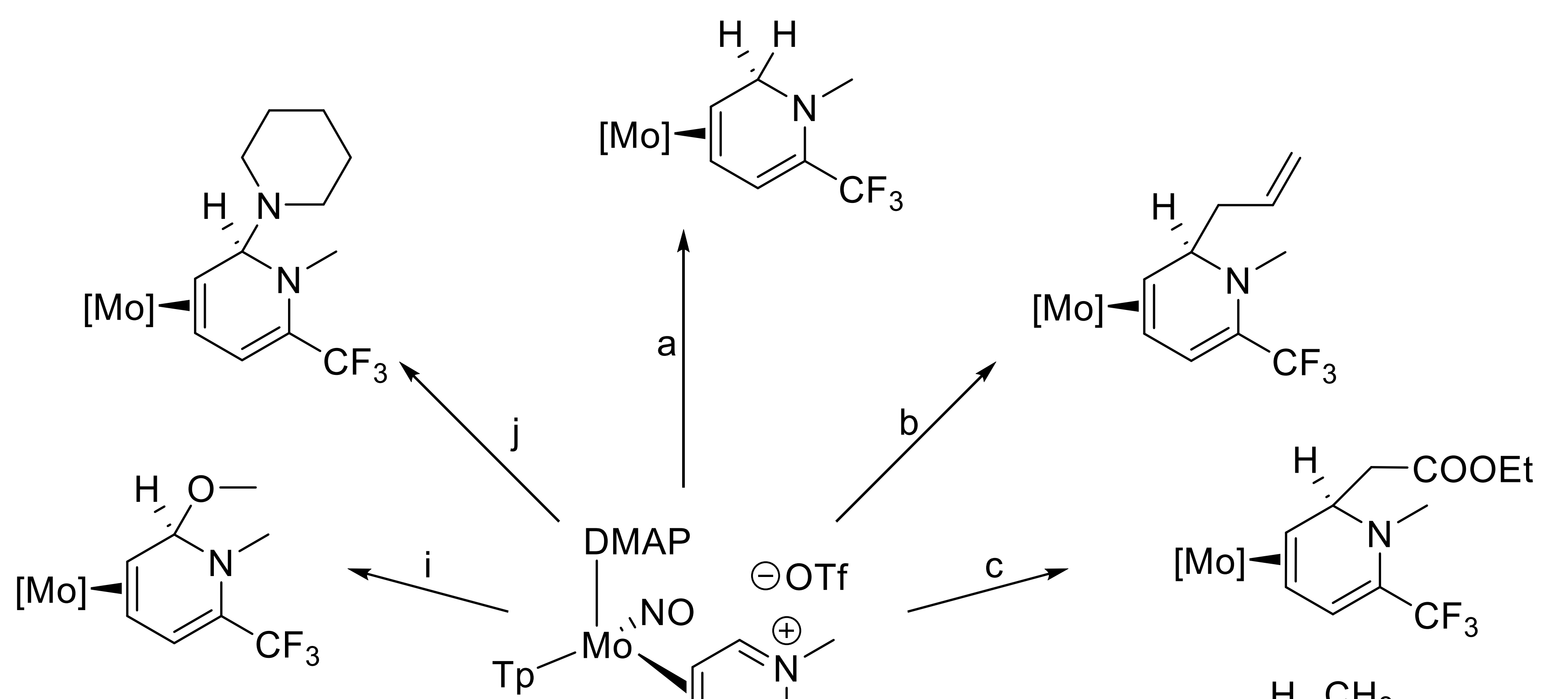
a: $\mathrm{KBH}_{4}, \mathrm{MeOH} ; \mathrm{b}: \mathrm{BrCH}_{2} \mathrm{CHCH}_{2}, \mathrm{Zn}, \mathrm{THF} ; \mathrm{c:} \mathrm{BrCH}_{2} \mathrm{CO}_{2} \mathrm{Et}, \mathrm{Zn}$, THF; d: $\mathrm{MeMgBr}^{\mathrm{T}} \mathrm{THF} ; \mathrm{e:} \mathrm{PhMgBr}$, THF; f:
3,4-(methylenedioxy)PhMgBr, THF; g:BnMgBr, THF; h: NaCN, MeCN; i: $\mathrm{NBu}_{4} \mathrm{OH}$, MeOH; j: piperidine

\section{Decomplexation/Elaboration}

- Organics can be freed from the metal by oxidation with ferrocenium salts or iodine

Method allows synthesis of non-aromatic derivatives that can be difficult to produce selectively by traditional organic chemistry

Liberated dihydropyridines can be elaborated into isoquinuclidines, a medicinallyrelevant scaffold found in alkaloids such as ibogaine

等,

$\int_{\mathrm{CN}}^{\mathrm{CN}} \sqrt{\mathrm{NC} \vee} \quad \mathrm{MeO}_{2} \mathrm{C} \vee \sqrt{ } \quad \mathrm{O}^{\mathrm{N}}=\mathrm{O}$ CN

$\mathrm{R}=\mathrm{Ph}, \mathrm{Bn}, \mathrm{p}-\mathrm{OCF}_{3} \mathrm{Bn}$, 3,4-(methylenedioxy)phenyl


Enantioselective Synthesis

- Enantioenrichment of metal complex retained though iodine oxidation and low temperature reduction in neat ligand

- Liberation of organic with iodine recycles metal complex

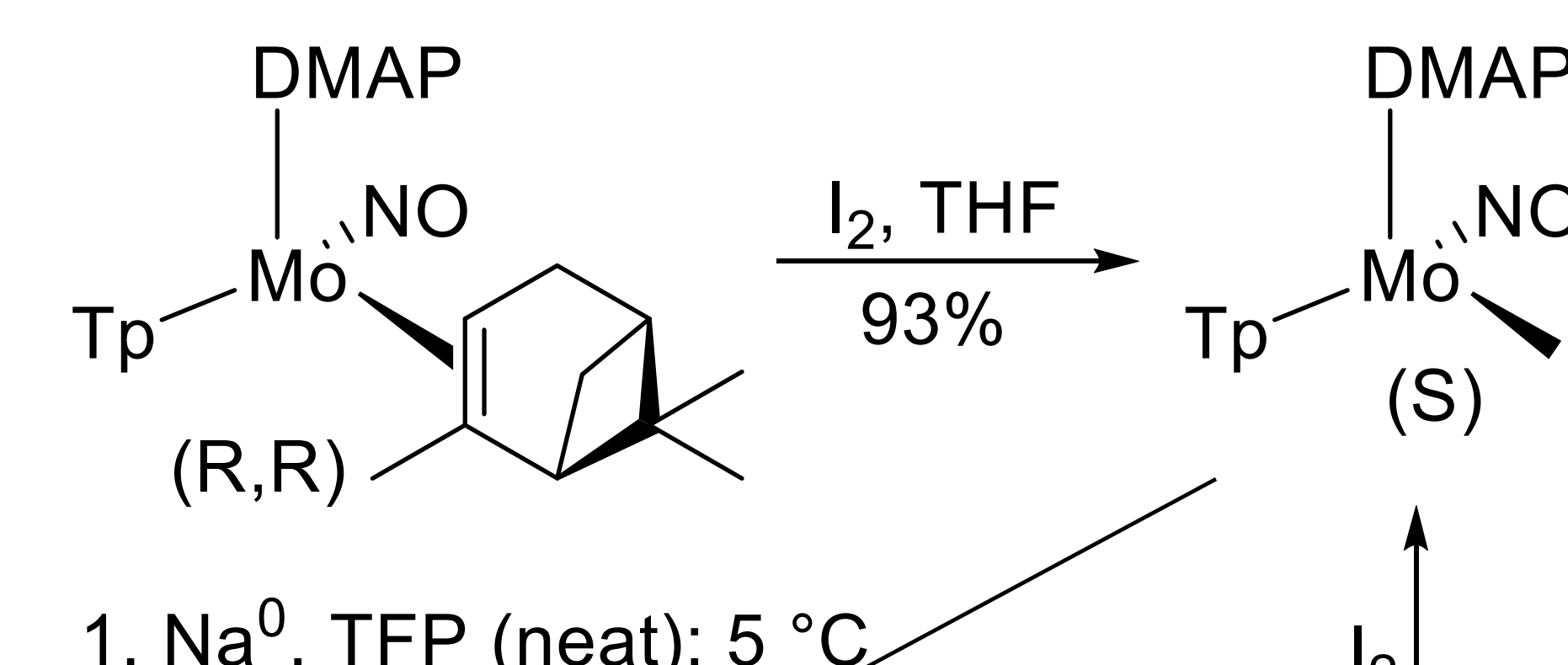

1. $\mathrm{Na}^{0}, \mathrm{TFP}\left(\right.$ neat); $5^{\circ} \mathrm{C}$

2. MeOTf, MeCN $24 \%$

$$
\text { (R) }
$$

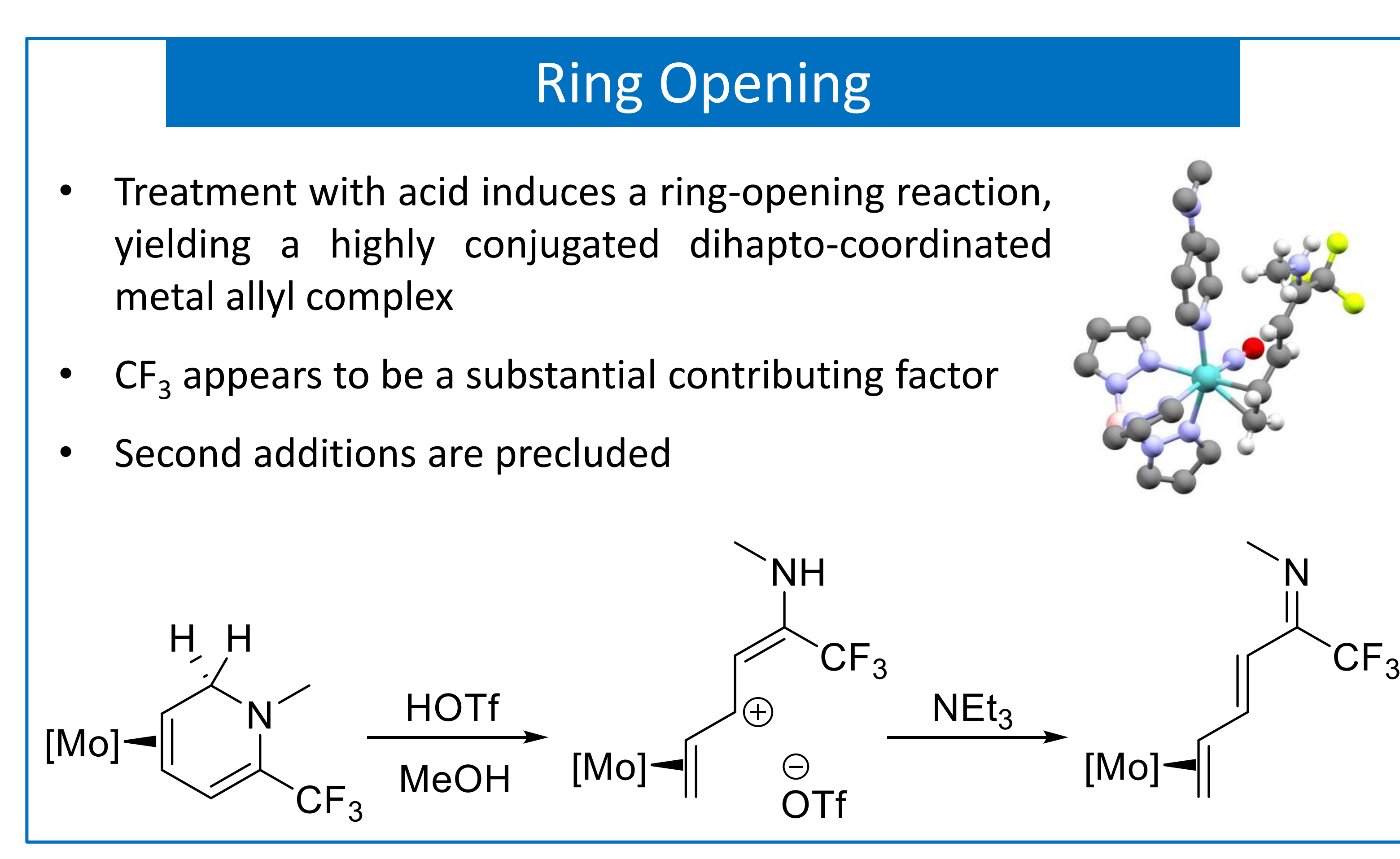

\section{Conclusions and Future Work}

- A wide range of $\eta^{2}$-coordinated pyridine complexes are accessible to molybdenum, avoiding $\mathrm{kN}$ coordination

Coordinated pyridines can be functionalized and subsequently decomplexed to produce dihydropyridines

Dihydropyridines can be elaborated into novel isoquinuclidines

- Enantioselective synthesis is possible, with demonstrated er > 94:6

- Conditions are being sought to functionalize remaining uncoordinated double bond of dihydropyridine complexes to yield tetrahydropyridines

\section{References}

1. Delafuente, D. A. et al., J. Am. Chem. Soc. 2007, 129 (2), 406-416. 2. Shivokevich, P. J. et al., Organometallics, 2018, 37 (23), pp 4446-4456. 3. Harrison, D. P. et al., J. Am. Chem. Soc. 2008, 130 (50), 16844-16845. 4. Harrison, D. P. et al., Organometallics 2009, 28 (19), 5682-5690. 5. Harrison, D. P. et al., J. Am. Chem. Soc. 2010, 132 (48), 17282-17295. 6. Pienkos, J. A. et al., Organometallics 2014, 33 (19), 5464-5469.

7. Myers, J. T. et al., Am. Chem. Soc. 2017, 139 (33), 11392-11400.

\section{Acknowledgements}

Dr. W. Dean Harman

- Dr. Diane A. Dickie

- Harman Lab

- Collaborators for biological testing

- NSF GRant (CHE-1152803)

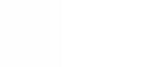

